\title{
Evolution of the new grapevine disease of Pinot gris and of Grapevine Pinot gris virus (GPGV)
}

\author{
Nadia Bertazzon, Vally Forte, Luisa Filippin, Michele Borgo, and Elisa Angelini \\ CREA Centro di Ricerca per la Viticoltura, 31015 Conegliano (Treviso), Italia
}

\begin{abstract}
An emergent grapevine disease, discovered about 10 year ago, has been associated to a new virus, called Grapevine Pinot gris virus (GPGV). The aim of the present work was to survey the presence of both the disease and the virus in Veneto (North Eastern Italy), and the presence of the virus in Europe in the last 15 years, using molecular techniques. The visual survey in Veneto showed that the disease is present in about $70 \%$ of the investigated vineyards, with a general low occurrence of symptomatic plants. However, the increase of the number of symptomatic grapevines in 8 much damaged vineyards was around $25 \%$ per year. The virus occurs in more than $80 \%$ of the plants. Finally, our data suggest that GPGV was limited to some Eastern European countries at least before 2005, and that it spread more widely throughout Europe after 2010.
\end{abstract}

\section{Introduzione}

Una malattia emergente della vite, caratterizzata da ritardo nel germogliamento, mosaicature e deformazioni fogliari e contrazione dello sviluppo vegetativo è stata identificata per la prima volta sulla cv. Pinot grigio in Nord Italia nel 2003 [1]. La patologia è stata successivamente osservata in varie regioni italiane e Paesi europei, come la Slovenia, anche su altre varietà [2-6]. Le cultivar più colpite finora sono Pinot grigio, Pinot bianco, Pinot nero, Gewürztraminer, Tocai friulano e Glera.

I sintomi, descritti soprattutto su Pinot grigio e Glera, sono più evidenti all'inizio della stagione vegetativa. Includono deformazioni fogliari, scolorimenti nervali e punteggiature delle foglie, nonché necrosi degli apici e ritardo nel germogliamento. Nei casi più gravi è stata riportata anche crescita stentata e progressiva perdita di vigoria.

Tale patologia causa un effetto depressivo sulle produzioni viticole. Dati preliminari ottenuti da viti malate delle cv. Pinot grigio, Gewürztraminer e Glera suggeriscono che $\mathrm{i}$ danni siano soprattutto di tipo quantitativo, con riduzione del peso dell'uva alla raccolta fino all' $80 \%$, dovuto alla diminuzione sia del numero di grappoli che del peso medio del grappolo $[7,8]$.

La malattia è stata associata alla presenza del Grapevine Pinot gris Virus (GPGV), un trichovirus isolato recentemente. Tale virus, dopo la sua scoperta, è stato ritrovato in diversi Paesi, come la Slovacchia e la Repubblica Ceca, la Francia, la Grecia, la Korea, la Cina, gli Stati Uniti ed il Canada [9-17]. GPGV però può infettare la vite anche senza causare alcun sintomo, rimanendo latente. Questo fatto sembra dipendere dalla presenza in loco di diverse varianti del virus, più o meno aggressive, dalla loro concentrazione, e probabilmente anche dalle condizioni agronomiche e climatiche $[6,18]$.

Lo scopo di questo lavoro è stato di verificare: 1) la presenza della sintomatologia e del virus nelle aree vitate del Veneto (Nord Est d'Italia), tramite il monitoraggio visuale e molecolare di circa 290 vigneti; 2) la presenza del virus in estratti di campioni di vite raccolti negli ultimi 15 anni da varie regioni Europee e conservati al CREA, tramite metodologie molecolari.

\section{Materiali e metodi}

\subsection{Rilievi in vigneto}

Nella primavera-estate del 2014 sono stati ispezionati 289 vigneti in alcuni territori Veneto (Nord-est Italia), coltivati principalmente con le cv. Glera e Pinot grigio. In ciascuno di questi vigneti sono state osservate e mappate le viti con sintomi della malattia, al fine di stimarne l'incidenza. Fra questi sono stati scelti 8 vigneti (Tabella 1), caratterizzati da un'alta presenza di piante sintomatiche, che sono stati monitorati visualmente per 3 anni consecutivi (20142016). Da circa metà dei vigneti sono stati raccolti campioni di foglie, subito congelati a $-80^{\circ} \mathrm{C}$, per la successiva analisi molecolare.

\subsection{Analisi molecolari}

Sono stati analizzati 150 campioni raccolti nel periodo 2013-2016, provenienti dai vigneti monitorati e dalle collezioni del CREA, con presenza o assenza di sintomi. Inoltre il virus è stato ricercato in estratti di RNA conservati nell'archivio del CREA a $-80^{\circ} \mathrm{C}$ e risalenti ad anni precedenti. In particolare, sono stati selezionati 75 campioni raccolti da germoplasma di vite coltivato in Veneto ed altri 102 provenienti da accessioni di vari Paesi Europei, raccolti negli anni 2002-2005. Poi, per confronto, le analisi molecolari sono state estese ad altri 114 campioni di vite di provenienza Europea, ma raccolti dopo il 2010. In totale, quindi, sono state analizzate 225 viti del Veneto e 216 di vari Paesi Europei.

Le analisi molecolari dei campioni sono state effettuate dopo avere estratto l'RNA secondo il protocollo di MacKenzie et al. [19]. Il cDNA è stato preparato secondo 
Tabella 1. Elenco e caratteristiche degli 8 vigneti osservati in Veneto per 3 anni consecutivi (2014-2016).

\begin{tabular}{|c|l|c|c|c|}
\hline $\mathbf{N}$ & Località & Cv & $\begin{array}{l}\text { Anno } \\
\text { impianto }\end{array}$ & $\begin{array}{l}\text { N tot viti } \\
\text { osservate }\end{array}$ \\
\hline 1 & $\begin{array}{l}\text { Refrontolo } \\
\text { (TV) }\end{array}$ & Glera & 2007 & 200 \\
\hline 2 & $\begin{array}{l}\text { Cison di Val- } \\
\text { marino(TV) }\end{array}$ & Glera & 1970 & 480 \\
\hline 3 & $\begin{array}{l}\text { Susegana } \\
\text { (TV) }\end{array}$ & Glera & 1990 & 900 \\
\hline 4 & $\begin{array}{l}\text { Refrontolo } \\
\text { (TV) }\end{array}$ & Glera & 2009 & 1500 \\
\hline 5 & $\begin{array}{l}\text { Ogliano } \\
\text { (TV) }\end{array}$ & Glera & 2012 & 625 \\
\hline 6 & $\begin{array}{l}\text { Jesolo } \\
\text { (VE) }\end{array}$ & Glera & 2012 & 2220 \\
\hline 7 & $\begin{array}{l}\text { Mansuè } \\
\text { (TV) }\end{array}$ & Traminer & 2008 & 1400 \\
\hline 8 & $\begin{array}{l}\text { Rua di Feletto } \\
\text { (TV) }\end{array}$ & Glera & 2011 & 2012 \\
\hline
\end{tabular}

la metodica descritta in Angelini et al. [20]. La diagnosi del virus GPGV è stata eseguita tramite l'uso di primer specifici per il virus, che amplificano una regione del gene del capside proteico di circa 430 bp (paia di basi) [15]. Sono inoltre stati usati dei primer di controllo che amplificano la regione del $18 \mathrm{~S}$ della vite, che servono per verificare la qualità dell'estratto, in particolare nel caso di campioni datati [21].

\section{Risultati e discussione}

\subsection{Rilievi in vigneto}

Nel 29\% circa (84 appezzamenti) dei vigneti osservati e coltivati con cultivar suscettibili non sono stati ritrovati sintomi ascrivibili alla malattia. Sul totale dei vigneti con sintomi (205 appezzamenti), la maggior parte manifesta un'incidenza molto bassa della malattia. Infatti, circa il $35 \%$ dei vigneti mostra un'incidenza di piante sintomatiche inferiore all' 1 per mille; il $50 \%$ dei vigneti presenta una percentuale di infezione fra l'1 per mille e '' $1 \%$; 1'11\% dei vigneti oggetto di monitoraggio si colloca fra 1 ' $1 \%$ e il $10 \%$ di infezione; il $4 \%$ dei vigneti invece manifesta un'incidenza di piante sintomatiche superiore al $10 \%$, con punte del $40 \%$.

Negli 8 vigneti scelti per il monitoraggio triennale dei sintomi è stato rilevato in genere un progressivo aumento del numero di piante sintomatiche, con incrementi annuali dal 3\% fino all'80\% di piante sintomatiche (Fig. 1). L'incremento medio fra il 2014 ed il 2015 e quello fra il 2015 ed il 2016 sono stati molto simili, attestandosi intorno al $25 \%$. L'intensità della sintomatologia è invece risultata maggiore in annate caratterizzate da piovosità elevate e temperature sotto la media in prossimità della ripresa vegetativa (2014 e 2016).

\subsection{Presenza del virus in Veneto}

La presenza del virus è stata rinvenuta in 117 campioni di vite sui 150 raccolti in Veneto fra il 2013 ed il 2016, con un'incidenza quindi del $78 \%$. Tale risultato è in linea con quanto trovato nelle altre due regioni italiane più colpite,

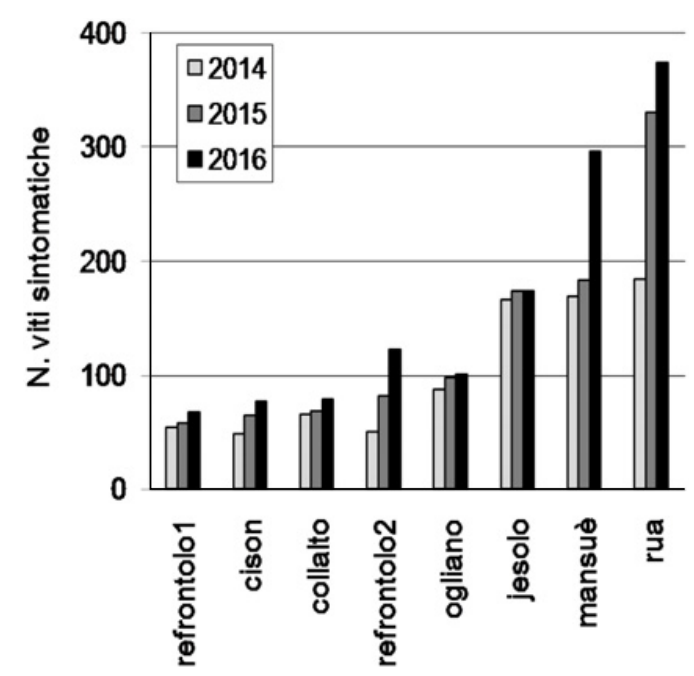

Figura 1. Numero di viti sintomatiche presenti nei 3 anni di osservazioni in campo negli 8 vigneti selezionati.

cioè il Trentino Alto Adige ed il Friuli Venezia Giulia, dove l'incidenza di GPGV nei vigneti è intorno all' $80 \%$ $[6,18]$. Tutte le viti sintomatiche sono risultate infette (33), mentre nelle viti senza sintomi GPGV era presente nel $72 \%$ dei casi (85 infetti su 117). Anche questo dato conferma risultati ottenuti precedentemente da altri autori, che segnalano come la presenza del virus spesso non sia associata alla manifestazione dei sintomi $[1,5,18]$.

Dopo aver verificato la qualità degli estratti conservati a $-80^{\circ} \mathrm{C}$ tramite l'amplificazione del gene endogeno di controllo, le analisi hanno evidenziato che fra i campioni di vite provenienti dal Veneto raccolti negli anni 2002-2005 solo una pianta di Cabernet franc era infetta, mentre tutte le altre 74 non contenevano il virus. Per confermare questo dato, 34 viti coltivate nelle collezioni ampelografiche del CREA di Conegliano, il cui estratto di RNA del 2002 era stato già analizzato, sono state campionate ed analizzate anche nel 2014: il 79,4\% di tali viti risultava infetto nel 2014. Questi risultati suggeriscono quindi che GPGV sia comparso recentemente in Veneto, e che si sia poi diffuso rapidamente nel territorio, anche nei vigneti più vecchi.

\subsection{Presenza del virus in Europa}

Fra i campioni di vite raccolti prima del 2005, sono risultati infetti quasi tutti quelli provenienti dalla Repubblica Ceca e dall'Ucraina, e circa metà di quelli provenienti dalla Macedonia e dal Montenegro (Tabella 2). Gli altri campioni, da Croazia, Francia, Grecia, Portogallo e Serbia sono risultati negativi (57), mentre 3 viti dalla Spagna, tutte della cultivar Garnacha, sono risultate infette.

Passando alle analisi su viti raccolte dopo il 2010, in media GPGV è stato rinvenuto su metà delle viti (62 su 114), anche nei Paesi dove non era stato identificato precedentemente. Sebbene il numero di campioni sia limitato, possiamo certamente evidenziare l'ampia diffusione del virus in Europa, che è stato ritrovato anche in Romania, Bosnia, Serbia, Croazia, Portogallo e Spagna. Questi dati sembrano quindi indicare che GPGV fosse limitato ad alcune regioni dell'Europa orientale almeno fino al 2005, e che poi si sia diffuso nel resto dei Paesi Europei, inclusa l'Italia. 
Tabella 2. Numero di viti risultate infette da GPGV rispetto al numero totale di viti analizzate. I dati sono presentati separatamente per il periodo precedente al 2005 e successivo al 2010, divisi per Paese Europeo da cui è stato raccolto il campione.

\begin{tabular}{|c|c|c|}
\hline \multirow{2}{*}{ Paese } & \multicolumn{2}{|c|}{ N. viti infette/N. viti analizzate } \\
\cline { 2 - 3 } & Prima del 2005 & Dopo il 2010 \\
\hline Bosnia & - & $1 / 15$ \\
\hline Croazia & $0 / 14$ & $10 / 16$ \\
\hline Repubblica Ceca & $16 / 17$ & - \\
\hline Francia & $0 / 8$ & $2 / 2$ \\
\hline Grecia & $0 / 11$ & $1 / 15$ \\
\hline Macedonia & $7 / 12$ & - \\
\hline Montenegro & $6 / 13$ & - \\
\hline Portogallo & $0 / 2$ & $7 / 16$ \\
\hline Romania & - & $16 / 16$ \\
\hline Serbia & $0 / 5$ & $1 / 3$ \\
\hline Spagna & $3 / 18$ & $8 / 15$ \\
\hline Ucraina & $2 / 2$ & $16 / 16$ \\
\hline $\begin{array}{c}\text { N. viti infette/ } \\
\text { N. totale viti } \\
\text { analizzate }\end{array}$ & $\mathbf{3 4 / 1 0 2}$ & $\mathbf{6 2 / 1 1 4}$ \\
\hline
\end{tabular}

A tal proposito vale la pena sottolineare che all'inizio degli anni 2000 nei principali Paesi Europei produttori di vino, quali Francia, Italia e Spagna, molti ricercatori e vivaisti, pubblici e privati, hanno importato diversi materiali viticoli dall'Est Europa, in cerca di caratteristiche interessanti quali la resistenza alle malattie, ma anche per scopi commerciali e di moltiplicazione. Dato che né la malattia né il virus erano a quel tempo conosciuti, e complice il fatto della latenza del virus, probabilmente materiali infetti da GPGV hanno avuto la possibilità di circolare liberamente nella Comunità Europea, favorendo la diffusione del virus sia per innesto che tramite vettore. Sembra infatti che il vettore del virus sia Colomerus vitis [22], l'acaro dell'erinosi, molto diffuso in Europa e contro il quale non viene generalmente effettuato alcun trattamento, poiché non arreca danni gravi alle piante in vigneto. Anche nell'Italia nord-orientale, dove la malattia ed il virus sono stati scoperti per la prima volta, $C$. vitis è molto comune in vigneto.

\section{Conclusioni}

Il lavoro dimostra che la malattia del Pinot grigio ed il virus associato sono molto diffusi in Veneto, ma anche in Europa. Le indagini hanno evidenziato come la situazione fitosanitaria si sia molto aggravata negli ultimi 10-15 anni, in quanto il virus non risultava presente in Veneto ed in moltissime aree viticole europee negli anni passati. Il quadro fitosanitario europeo richiede però ulteriori conferme, che si possono ottenere solo tramite monitoraggi puntuali nei diversi Paesi. La sintomatologia in vigneto è anch'essa in aumento, con un incremento annuale di piante sintomatiche stimato intorno al $25 \%$ per il Veneto.

La situazione è quindi seria. Mancano ancora molte informazioni scientifiche e tecniche su tale patologia, in particolare sul rapporto fra virus e malattia, sulle interazioni con l'ambiente e le diverse cultivar, sulle possibili modalità di prevenzione, lotta e controllo. Data la presenza del virus anche in alcuni Paesi extraeuropei, e le problematiche di esportazione nei Paesi ancora immuni, si auspica uno sforzo collettivo della comunità scientifica per affrontare in modo adeguato tale problematica emergente, che ormai ha assunto dimensioni mondiali.

\section{Bibliografia}

[1] A. Giampetruzzi, V. Roumi, R. Roberto, U. Malossini, N. Yoshikawa, P. La Notte, F. Terlizzi, R. Credi, P. Saldarelli, Virus Res. 163, 262 (2012)

[2] R. Beber, A.R. Babini, F. Terlizzi, C. Poggi Pollini, R. Credi, C. Ratti, J Plant Pathol. 95, S4.36 (2013)

[3] A. Raiola, C. Scopel, D. Ferrigo, F. Taglietti, C. Duso, R. Causin, J. Plant Pathol. 95, S4.58 (2013)

[4] P. Casati, G. Durante, F. Quaglino, E. Zacchi, P.A. Bianco, Proceedings of the $X X$ Congress of the Italian Phytopathological Society, 62 (2014)

[5] I. Mavrič Pleško, V.K. Marn, G. Seljak, I. Zezlina, Plant Dis. 98, 1014 (2014)

[6] G.L. Bianchi, F. De Amicis, L. De Sabbata, et al., EPPO Bull 45, 22 (2015)

[7] U. Malossini, R. Moscon, M. Ferrazza, P. Bianchedi, M. Varner, R Credi, Atti IV Convegno Nazionale di Viticoltura, 37 (2012)

[8] N. Bertazzon, V. Forte, I. Bazzo, L. Filippin, E. Angelini, L'Inf. agrario 9, 66 (2015)

[9] I.S. Cho, S.M. Jung, J.D. Cho, G.S. Choi, H.S. Lim, New Dis. Rep. 27, 10 (2013)

[10] M. Glasa, L. Predajňa, P. Komínek, A. Nagyová, T. Candresse, A. Olmos, Arch. Virol. 159, 2103 (2014)

[11] M. Beuve, T. Candresse, M. Tannières, O. Lemaire, Dis Notes 99, 293 (2015)

[12] G.P. Martelli, J. Plant Pathol. 96, S105 (2014)

[13] M. Al Rwahnih, D. Golino, A. Rowhani, Dis. Notes 100, 1030 (2016)

[14] H. Xiao, M. Shabanian, W. McFadden-Smith, B. Meng, Dis. Notes 100, 1030 (2016)

[15] E. Angelini, N. Bertazzon, J. Montgomery, X. Wang, A. Zinkl, J. Stamp, Dis. Notes 100, 1254 (2016)

[16] X.D. Fan Y.F. Dong, Z.P. Zhang, F. Ren, G.J. Hu, Z.N. Li, J. Zhou, Dis. Notes 100, 540 (2016)

[17] S. Poojari, T. Lowery, M. Rott, A.M. Schmidt, J.R. Úrbez-Torres, Dis. Notes 100, 1513 (2016)

[18] P. Saldarelli, A. Giampetruzzi, M. Morelli, U. Malossini, C. Pirolo, P. Bianchedi, V. Gualandri, Phytopathol. 105, 555-63 (2014)

[19] D.J. MacKenzie, M.A. McLean, S. Mukerji, M. Green, Plant Dis. 81, 222 (1997)

[20] E. Angelini, N. Bertazzon, M. Borgo, J. Phytopathol. 152, 416 (2004)

[21] G. Gambino, I. Gribaudo, Phytopathol. 96, 1223 (2006)

[22] V. Malagnini, E. De Lillo, P. Saldarelli, et al., Proceedings of the 18th Meeting of the ICVG, 217 (2015) 